\title{
The Nature of Dark Energy
}

\author{
Luca Amendola, Claudia Quercellini, Domenico Tocchini-Valentini, \\ and Alessandro Pasqui \\ INAF - Observatorio Astronomico di Roma, \\ Via Frascati 33, 00040 Monte Porzio Catone, Italia
}

Received on 23 May, 2001

\begin{abstract}
According to a variety of cosmological observations at small and large redshifts, the universe is composed by a large fraction of a weakly clustered component with negative pressure, called dark energy. The nature of the dark energy, i.e. its interaction and self-interaction properties, is still largely unknown. In this contribution we review the properties of dark energy as inferred from observations, with particular emphasis on the cosmic microwave background. We argue that the current dataset imposes strong constraints on the coupling of dark energy to dark matter, while it is still insufficient to constrain the equation of state or potential. Future data will dramatically improve the prospects.
\end{abstract}

\section{Rods, clocks and candles}

Four years after the first observational hints from the supernovae Ia (SNIa) Hubble diagram about the existence of a dominant component of unclustered matter with negative pressure (Riess et al. 1998, Perlmutter et al. 1999), the so-called dark energy or quintessence (Wetterich 1988; Ratra and Peebles 1988; Frieman et al. 1995; Caldwell et al. 1998), there are still very few indications as to its nature. The main reason, perhaps, is that we lack any specific theoretical suggestion on the properties of the dark energy, i.e. on its selfinteraction potential and on how it interacts with the other cosmological components. At this stage, all we can do is to explore a wide range of phenomenological models specified by the potential and the coupling to the other fields in order to provide an overall best fit to the current data. Fortunately, there are now several cosmological observations that are at least potentially able to put stringent constraints on the nature of dark energy.

As with any other fundamental field, the dark energy can be characterized by how it interacts with itself and with the other fields. In other words, it can be characterized by its potential and couplings. While there have been several papers that tried to constrain the potential or the equation of state of the dark energy (Amendola 2001, Baccigalupi et al. 2001, Corasaniti and Copeland 2001, Bean and Melchiorri 2001), the study of its coupling has been relatively scarce, although it has been suggested several times that we cannot expect a vanishing coupling (Carroll 1998).

The observations that can reveal dark energy are varied, and can be classified into those that take into account only the evolution of the homogeneous background and those that investigate the growth of perturbations. The latter class is so far still poor of results, due to the large errors in the observations of the growth of clustering, although the prospects are interesting (Newman et al. 2001). Moreover, the perturbation growth is very similar for all models of dark energy, since the acceleration stops the gravitational instability for all but one model of dark energy. The single case in which this is not true has been discussed in Amendola and Tocchini-Valentini (2002).

The background observations all reduce essentially to the use of a standard measure, either candles, rods or clocks: any of this standard is in fact subject to a geometric apparent variation when seen at high redshift that depend on cosmology. The standard candles have been employed in the method based on the supernovae Ia, while standard rods are assumed in the AlcockPaczinsky method (or rather, a standard isotropy, see e.g. Calvao et al. 2001), in the lensing statistics (e.g. Cooray and Huterer 1999, Giovi et al. 2001 ) and in the size of the sound horizon at decoupling as detected from the CMB acoustic peaks (see e.g. Doran et al. 2000). Finally, standard clocks are employed in the method based on the galaxy ages (Alcaniz and Lima 2001, Jimenez and Loeb 2001). They all rely on the fact that the proper distance to an object at redshift $z$ depends on cosmology:

$$
S[r(z)]=\int_{0}^{z} \frac{d z^{\prime}}{E\left(z^{\prime}\right)}
$$

where the function $S[x]$ is $\left|\Omega_{k}\right|^{-1 / 2} \sin ^{-1}\left(\left|\Omega_{k}\right|^{1 / 2} x\right), x$, $\left|\Omega_{k}\right|^{-1 / 2} \sinh ^{-1}\left(\left|\Omega_{k}\right|^{1 / 2} x\right)$ depending on the spatial curvature $k=1,0,-1$, respectively, and where from the 
Friedmann equation

$$
\begin{aligned}
H^{2}(z) & =H_{0}^{2} E(z)^{2} \\
E^{2}(z) & =\Omega_{m}(1+z)^{3}+\Omega_{d e}(1+z)^{3 w_{d e}}+\Omega_{k}(1+z)^{2} .
\end{aligned}
$$

The subscripts refer to matter $(m)$, dark energy (de) and curvature $(k)$, and $w_{d e}$ is the dark energy parameter of state, $p_{d e}=\left(w_{d e}-1\right) \rho_{d e}$ and by definition $\Omega_{m}+\Omega_{d e}+\Omega_{k}=1$. It is clear therefore that all quantities that depend on distance, like luminosity, angular sizes, ages, also depend on cosmology. For instance, the method based on the SN Ia Hubble diagram determines the distance to a supernova (whose absolute magnitude $M$ has been inferred and corrected by local observations), via the distance modulus

$$
m-M=5 \log d_{L}(z)+25
$$

(plus possibly a $K$-correction) and then compares this value to the theoretical luminosity distance $d_{L}(z)=$ $r(z)(1+z)$ to find the best fit in terms of the parameters $H_{0}, \Omega_{m}, \Omega_{d e}, w_{d e}$. The case $w_{d e}=0$ corresponds to a pure cosmological constant model.

The main advantages with this approach are that the dependence on the cosmological constant is simple and that one can observe $r(z)$ at different redshifts, depending on the object. So far, SN Ia have been seen up to $z \approx 1$ (with a couple of SN beyond this), but future experiments can extend the observations up to $z \approx 2$ and to a much extended dataset. Moreover, the Alcock-Paczyinsky method can be applied to quasars at $z \approx 5$ or beyond. The distance of the sound horizon at decoupling, expressed as the angular diameter distance $d_{A}(z)=r(z) /(1+z)$, in turn, reaches $z \approx 1000$, the redshift of the last scattering surface. The crucial point is that $r(z)$ depends on the parameters in a different way for different redshifts. In Fig. 1 we show the lines of constant $d_{L}(z)$ for a pure $\Lambda$ model assuming the sources are at $z=0.7,4,10,1000$ : the contours follow a different orientation, rotating anticlockwise for increasing $z$, being almost vertical for $z \approx 4$. Therefore, observations at low redshift, like the SNIa, and at high redshift, like the sound horizon at decoupling, can give orthogonal constraints, thereby determining with high precision the cosmological parameters.

The observation of the CMB fluctuation spectrum fall in between the two classes, since the spectrum depends both on the background and on the perturbations: in fact, at small angular scales the spectrum is mostly determined by the angular-diameter distance to decoupling, while at large angular scales it depends on the growth of the gravitational potential through the integrated Sachs-Wolfe effect and on the intrinsic fluctuations of the dark energy field. In this review I will focus mostly on the costraints from the recent high resolution CMB data (Netterfield et al. 2001, Lee et al. 2001, Halverson et al. 2001) because they appear to be the most powerful and with the highest chance of rapid development. The reason is that they are not subject to the theoretical uncertainties that still enshroud the SNIa method. In fact, in contrast to the SNIa, the physics of the CMB fluctuations is relatively well understood, and there are no problems of evolution. Moreover, experiments are underway that should expand the statistics by a factor of a hundred or more in a few years.
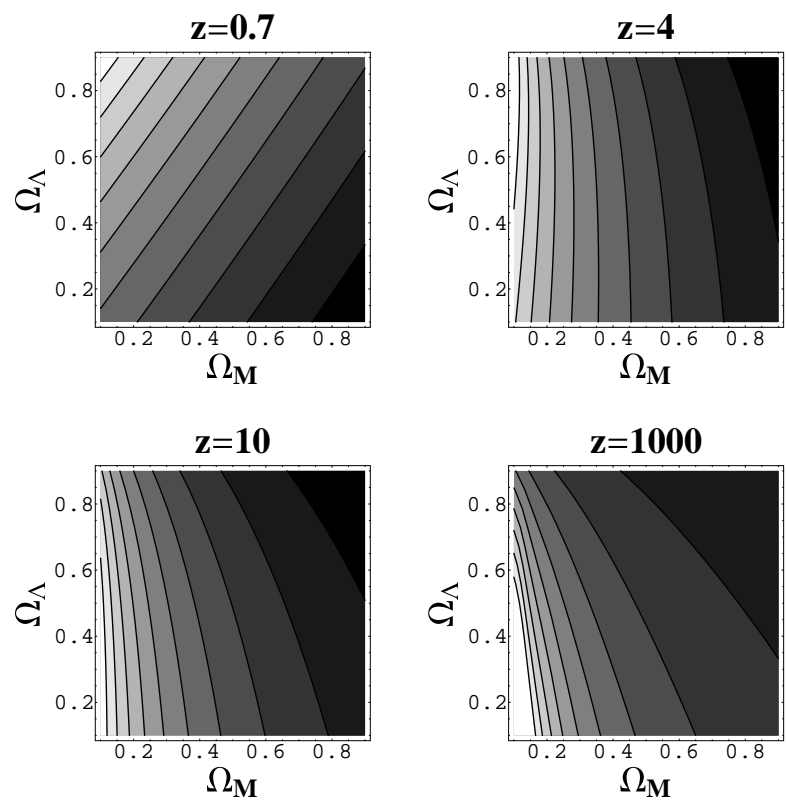

Figure 1. Contours of constant luminosity distance $d_{L}(z)$ for various values of $z$. At small redshift the lines follow approximately the relation $\Omega_{m}-\Omega_{\Lambda}=$ const, and at large redshift the orthogonal relation $\Omega_{m}+\Omega_{\Lambda}=$ const .

Several works have tried to constrain dark energy models with the recent CMB data, leading in some cases to interesting but conflicting results. In Amendola (2001) the dimensionless coupling parameter $\beta$ that measures the strength of the interaction of dark energy to dark matter with respect to the gravitational interaction was constrained to be smaller than 0.1 roughly, adopting an exponential potential. The slope of the potential, on the other hand, was found to be essentially unconstrained by the data. Baccigalupi et al. (2002) found that, among power-law potentials $V \sim \phi^{-\alpha}$ and fixing the Hubble constant to $h=0.65$ (in units of $100 \mathrm{~km} / \mathrm{sec} / \mathrm{Mpc}$ ), values of $\alpha$ around unity are favored while the case of pure cosmological constant, $\alpha=0$, was less likely by a factor of five roughly. Corasaniti and Copeland (2001), on the other hand, have shown that an equation of state close to that of a pure cosmological constant gives the best fit to the data, particularly for as concerns the position of the acoustic peaks. Bean and Melchiorri (2002) also conclude that the pure cosmological constant gives the best fit to the CMB data when the prior on the Hubble constant is broadened to $h=0.72 \pm 0.08$.

Recently, we extended the previous studies in two respects. First, we constrained not only the dark energy self-interaction (i.e. its potential) but also its in- 
teraction with matter. Second, we put only very weak restrictions on the cosmological parameters $h, \Omega_{b, c}$ (the density parameters of baryons and cold dark matter, respectively), and $n_{s}$ (the slope of the scalar perturbations). The conflicting results above are in fact due mostly to different priors.

These generalizations allowed us to find that the present CMB data are capable to put a strong constraint on the coupling but not on the scalar field potential or equation of state. In fact, the degeneracy between $h$ and $w_{\phi}$, the dark energy equation of state (Huey et al. 1999) almost erases the sensitivity of the CMB to the dark energy potential. In sharp contrast, the CMB spectra are very sensitive to the dark energy coupling, since the latter determines the equation of state for a long stage after equivalence and there are no strong degeneracies with the other cosmological parameters.

\section{A Dark-dark coupling}

Let us consider two components, a scalar field $\phi$ and CDM, described by the energy-momentum tensors $T_{\mu \nu(\phi)}$ and $T_{\mu \nu(c)}$, respectively. General covariance requires the conservation of their sum, so that it is possible to consider an interaction between dark energy and dark matter such that

$$
\begin{aligned}
T_{\nu(\phi) ; \mu}^{\mu} & =C T_{(c)} \phi_{; \nu}, \\
T_{\nu(c) ; \mu}^{\mu} & =-C T_{(c)} \phi_{; \nu} .
\end{aligned}
$$

Such a coupling can be obtained from the conformal transformation of a Brans-Dicke gravity (see e.g. Amendola 1999) and it has been considered several times in literature starting from Wetterich $(1988,1995)$ and Wands et al. (1993). It has been discussed in the context of dark energy models in Amendola $(1999,2000)$ and, in a list which is meant to be only representative, Wands and Holden (2000), Chimento et al. (2000), Billyard and Coley (2000), Chiba (2001), Albrecht et al. (2001), Esposito-Farese and Polarsky (2001). In its conformally related Brans-Dicke form has been studied by Uzan (1999), Chiba (1999), Chen and Kamionkowsky (1999),Batista et al. (2000), Bertolami and Martins (2000), Baccigalupi et al. (2000), Faraoni (2000), Sen and Sen (2001). Theoretical motivations in superstring models and in brane cosmology have been proposed recently in Gasperini, Piazza and Veneziano (2002) and
Pietroni (2002). Other authors considered a coupling to specific standard model fields rather than to a generic form of dark matter: Carroll (1998) to the electromagnetic field, Horvat (2000) to neutrinos, Li et al. (2002) to baryonic or leptonic current: in these cases, there are strong constraints from local observations or from variation of fundamental constants, see e.g. Damour et al. (2002). In contrast, a coupling to dark matter is observable only with cosmological experiments, involving growth of perturbations or global geometric effects (proper distance).

In the flat conformal FRW metric $d s^{2}=a^{2}\left(-d \tau^{2}+\right.$ $\left.\delta_{i j} d x^{i} d x^{j}\right)$ the scalar field and dark matter conservation equations are

$$
\begin{aligned}
\ddot{\phi}+2 H \dot{\phi}+a^{2} U_{, \phi} & =C \rho_{c} a^{2}, \\
\dot{\rho}_{c}+3 H \rho_{c} & =-C \rho_{c} \dot{\phi}
\end{aligned}
$$

(dots refer to conformal time) where $H=\dot{a} / a$. We suppose now that the potential $U(\phi)$ is specified by the following relation

$$
U^{\prime}=B U^{N}
$$

(the prime refers to derivation with respect to $\phi$ ): this form includes power laws, exponential potentials and a pure cosmological constant, i.e. the most common forms of dark energy potentials. In the case of power law potential $U=A \phi^{-\alpha}$ we have

$$
N=(1+\alpha) / \alpha
$$

and $B=-\alpha A^{(-1 / \alpha)}$, while for the exponential potential $N=1$. We consider only the range $N \geq 1$ since for negative $\alpha$ there are no asymptotically accelerating models; for $N \rightarrow \infty$ we recover the pure cosmological constant. Clearly, for $A=0$ the potential vanishes, and we reduce to a theory which is conformally equivalent to pure Brans-Dicke gravity.

Assuming that the baryons are not directly coupled to the dark energy (otherwise local gravity experiment would reveal a fifth force, see Damour et al. 1990) and that the radiation as well is uncoupled (as it occurs if the coupling is derived by a Brans-Dicke Lagrangian, see e.g. Amendola 1999), the system of one Einstein equation and four conservation equations (for radiation, $\gamma$, baryons, $b$, CDM , $c$, and scalar field) can be conveniently written introducing the following five variables that generalize Copeland et al. (1997):

$$
x=\frac{\kappa}{H} \frac{\dot{\phi}}{\sqrt{6}}, \quad y=\frac{\kappa a}{H} \sqrt{\frac{U}{3}}, \quad z=\frac{\kappa a}{H} \sqrt{\frac{\rho_{\gamma}}{3}}, \quad v=\frac{\kappa a}{H} \sqrt{\frac{\rho_{b}}{3}}, \quad w=\frac{H}{a}
$$

where $\kappa^{2}=8 \pi G$ (notice that $w=d \log a / d t$ i.e. the usual Hubble constant). Notice that $x^{2}, y^{2}, z^{2}$, etc. correspond to the density parameter of each component. In terms of the independent variable $\log a$ we have then the system:

$$
x^{\prime}=\left(\frac{z^{\prime}}{z}-1\right) x-\mu y^{2 N} w^{2 N-2}+\beta\left(1-x^{2}-y^{2}-z^{2}-v^{2}\right),
$$




$$
\begin{aligned}
y^{\prime} & =\mu x y^{2 N-1} w^{2 N-2}+y\left(2+\frac{z^{\prime}}{z}\right), \\
z^{\prime} & =-\frac{z}{2}\left(1-3 x^{2}+3 y^{2}-z^{2}\right), \\
v^{\prime} & =-\frac{v}{2}\left(-3 x^{2}+3 y^{2}-z^{2}\right) \\
w^{\prime} & =-\frac{w}{2}\left(3+3 x^{2}-3 y^{2}+z^{2}\right)
\end{aligned}
$$

where

$$
\beta=C \sqrt{\frac{3}{2 \kappa^{2}}}, \quad \mu=3^{N} \kappa^{1-2 N} \frac{B}{\sqrt{6}} .
$$

The dimensionless constant $\beta^{2}$ can be seen as the ratio of the dark energy-dark matter interaction with respect to gravity. It can be shown in fact (Damour and Nordtvedt 1993, Wetterich 1995) that the force acting between dark matter particles can be described in the Newtonian limit as a renormalized Newton's constant $\hat{G}=G\left(1+4 \beta^{2} / 3\right)$. This shows that, in terms of the "post-Einsteinian" parameter $\eta$ (see Groom et al. 2001) which characterizes the deviation from General Relativity, we have $\eta=4 \beta^{2} / 3$. The effect of this interaction on structure growth is discussed in Amendola and Tocchini-Valentini (2002).

The system (8) includes several qualitatively different behaviors, already discussed in Amendola (2000), Tocchini-Valentini and Amendola (2002). However, for the range of values that are of cosmological interest, the system passes through three distinct phases after equivalence.

$\phi \mathrm{MDE}$. Immediately after equivalence, the system enters a matter dominated epoch with a non-negligible $\phi$ contribution, that we denote $\phi \mathrm{MDE}$, in which the dark energy potential density is negligible while the kinetic energy density parameter $\Omega_{K \phi}=x^{2}$ of the scalar field gives a constant contribution to the total density. As will be shown in the following, the existence of such an epoch is crucial for the constraints that we will be able to put on the coupling. It is not difficult to see that, neglecting radiation and baryons, the point $y=0$, $x=2 \beta / 3$ is a saddle point solution of the system (8) for any $N$ and for $|\beta|<\sqrt{3} / 2$. The system stays on the $\phi \mathrm{MDE}$ solution until $y$ starts growing. Along this solution the scale factor expands slower than a pure MDE, i.e. as

$$
a \sim t^{4 /\left(6+4 \beta^{2}\right)} .
$$

Since $y=0$ on the $\phi \mathrm{MDE}$, its existence is independent of the potential, although it has to verified for each potential whether it is a saddle.

Tracking trajectories. Let us now neglect baryons and radiation and put $\beta=0$. The tracking solutions found in Steinhardt et al. (1999) assume $y / x=p$ and $y^{2 N-1} w^{2 N-2}=q$ where $p, q$ are two motion integrals. In the limit $y^{2}, x^{2} \ll 1$ it is easy to show that $p^{\prime}=q^{\prime}=0$ if

$$
p^{2}=4 N-3
$$

$$
q=-\frac{3 p}{2 \mu(2 N-1)}
$$

The same tracking behavior remains a good approximation for small $\beta$. For $N=1$ the tracking solution becomes actually a global attractor of the dynamical system, see below. In the other cases, the tracking interpolates between the $\phi \mathrm{MDE}$ and the global attractor.

Global attractors. In the system (8) for $N \neq 1$ there exists only the attractor $x=0, y=1$ on which the dark energy completely accounts for the matter content. For $N=1$ the phase space is much richer, and there are several possible global attractors, only two of which accelerated (Amendola 2000). One, for $\mu>\left(-\beta+\sqrt{18+\beta^{2}}\right) / 2$, presents a constant non-zero $\Omega_{c}$ : this attractor actually coincides with the tracking solutions and in fact realizes the condition $p=1$ and $y=-3 /(2 \mu)$ as requested by Eq. (11) for $\beta=0$. This "stationary attractor" can be accelerated if $\beta>2 \mu$ and could solve the coincidence problem since $\Omega_{c} \propto \Omega_{\phi}$; it has been discussed in detail in Amendola and TocchiniValentini $(2001,2002)$. The other accelerated attractor occurs when $\mu<\left(-\beta+\sqrt{18+\beta^{2}}\right) / 2$ : in this case there are no tracking solutions and the global attractor reduces to $x=0, y=1$ as for $N \neq 1$. These solutions have already been compared to CMB for $N=1$ in Amendola (2001). The inclusion of the baryons modifies the considerations above but, as long as they are much smaller than the other components, the qualitative behavior of the system remains the same. It is to be noticed that the final attractor, on which the dark energy dominates completely the cosmic fluid, is yet to be reached, and therefore the existence of an accelerated epoch at the present depends mostly on the tracking.

The existence of the $\phi \mathrm{MDE}$ saddle and of the tracking solutions is crucial for our analysis. In fact, these two epochs guarantee that the equation of state of the scalar field is piece-wise constant through essentially all the post-equivalence epoch. In the $\phi \mathrm{MDE}$ phase the effective parameter of state $w_{e}=p_{t o t} / \rho_{t o t}+1$ and the field equation of state $w_{\phi}=p_{\phi} / \rho_{\phi}+1$ are

$$
w_{e}=1+\frac{4}{9} \beta^{2}, \quad w_{\phi}=2
$$

while during the tracking phase

$$
w_{e} \approx 1, \quad w_{\phi} \approx \frac{2}{1+p^{2}}=\frac{1}{2 N-1}
$$


(the last relation is approximated and it is actually only an upper bound to the present $w_{\phi}$; it is more precise if $w_{\phi}$ is identified with the average equation of state after $\phi \mathrm{MDE}$ rather than the present equation of state). Therefore, the cosmic evolution depends on $\beta$ alone during the $\phi \mathrm{MDE}$, and on $N$ alone during the tracking. Since the position of the acoustic peaks is related to the equation of state through the angular diameter distance, it appears that the CMB is able to put direct constraints on both $\beta$ and $N$. Since the sign of $\beta$ is ininfluent, we confine ourselves hereinafter to $\beta>0$. In Fig. 2 we show a typical trajectory that presents in sequence the three epochs discussed above. In Fig. 3 we present a three-dimensional phase space $x, y, z$ (i.e., field kinetic energy, potential energy, and radiation energy, respectively) for $N=1$, neglecting baryons (the equation for $w$ decouples for $N=1$ ). Notice the location of the $\phi \mathrm{MDE}$ and the final attractor.
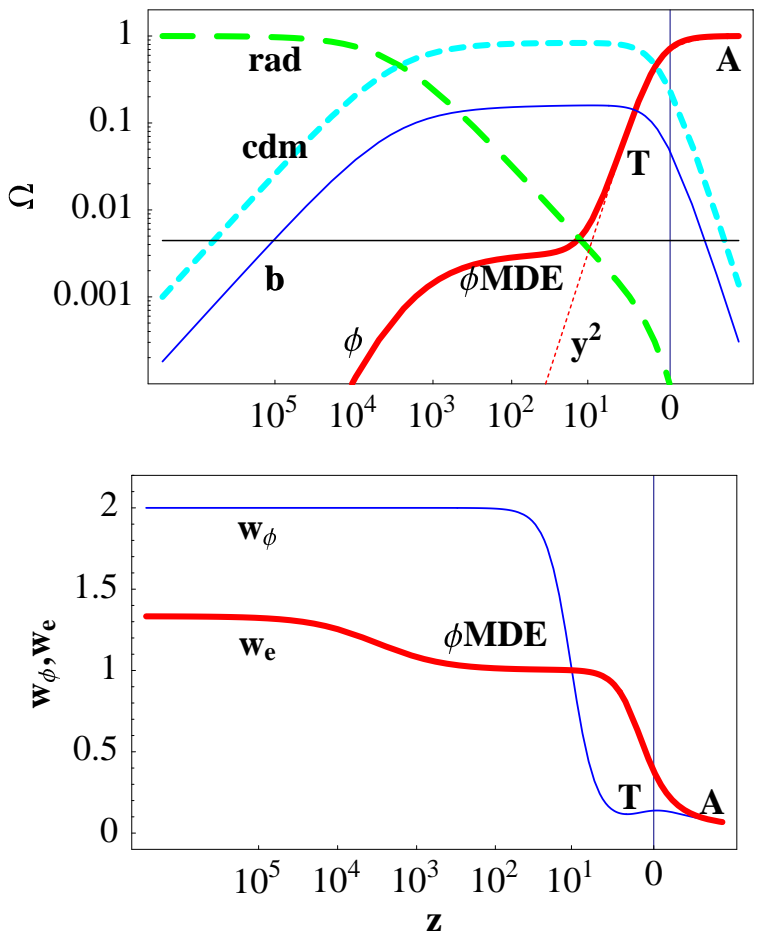

Figure 2. Numerical solutions of the system (8) for $N=$ $2, \beta=0.1, \omega_{c}=0.1, \omega_{b}=0.02, h=0.65$ plotted against the redshift. Upper panel. Long dashed line: radiation; short dashed line: CDM; unbroken thin line: baryons; unbroken thick line: scalar field; dotted line: scalar field potential energy. The horizontal thin line marks the kinetic energy density of $\phi \mathrm{MDE}$, reached just after equivalence. Lower panel. Thick line: effective equation of state; thin line: dark energy equation of state. The labels mark the $\phi \mathrm{MDE}$, the tracking $(\mathrm{T})$ and the final attractor $(\mathrm{A})$.

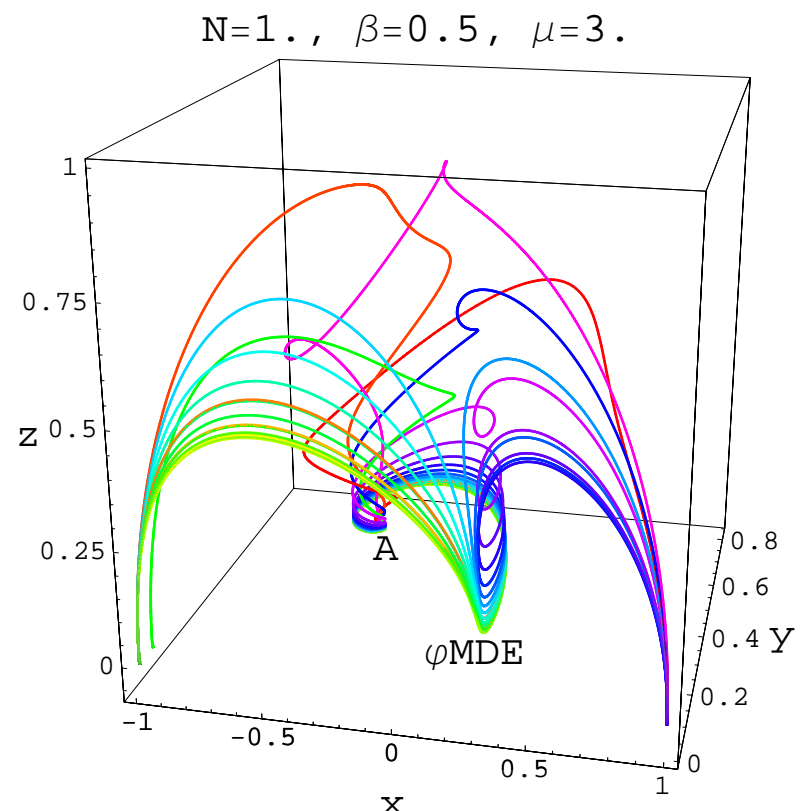

Figure 3. Three-dimensional phase space for $N=1$. The trajectories start at $x= \pm 1$. They all end at the global attractor, but most pass through the $\phi \mathrm{MDE}$ saddle.

There is however a crucial difference between the $\phi \mathrm{MDE}$ and a tracking for what concerns here: while the present dark energy equation of state, set by the tracking, is degenerated with $h$ for as concerns the CMB spectrum (see e.g. Huey et al. 1999; Bean and Melchiorri 2002), the equation of state during the $\phi \mathrm{MDE}$ is not. In fact, the angular diameter distance to the last scattering surface $d_{A}$ is degenerate along lines $h\left(w_{\phi}\right)$ for which

$$
d_{A} \sim \int_{a_{d e c}}^{1} d a\left[\omega_{c} a+\left(h^{2}-\omega_{c}\right) a^{4-3 w_{\phi}}\right]^{-1 / 2}=\text { const }
$$

where $\omega_{c} \equiv \Omega_{c} h^{2}$ (although this is exact only for $\beta=0$ it remains a good approximation even for small nonzero values). In Fig. 4 the degeneracy between spectra for different values of $h$ and $N$ (the other parameters being equal) appears clear. Assuming a strong prior on $h$ a peak emerges in the likelihood for $N$ but then the result is clearly prior-dependent. The same holds true for models in which the equation of state is slowly varying (see e.g. Huey et al. 1999, Doran et al. 2001). On the other hand, the fact that $\Omega_{\phi} \neq 0$ at decoupling in coupled models implies that the effects of the coupling on the CMB are not due solely to the angular diameter distance, and therefore the geometric degeneracy can be broken. This is shown in Fig. 5 in which $C_{\ell}$ spectra for various values of $\beta$ (all other parameters being equal) are shown: the spectra change both in amplitude and in peak's position. 


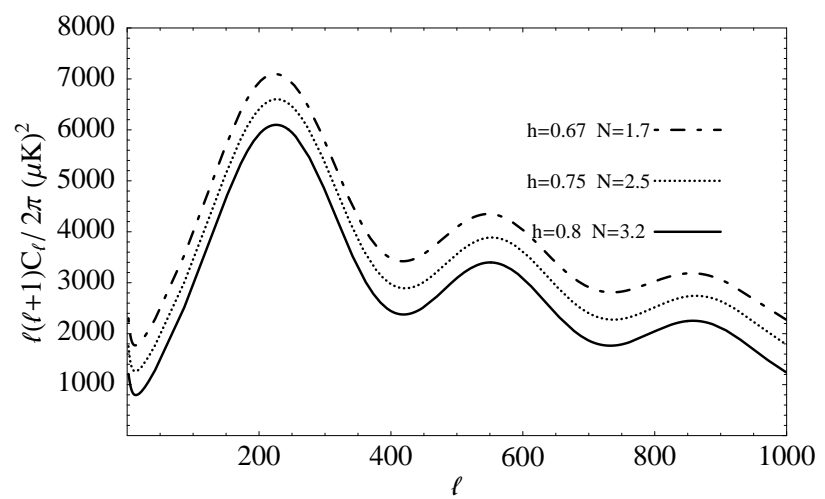

Figure 4. Spectra for degenerated parameters (the other parameters are $\beta=0, \omega_{b}=0.01, \omega_{c}=0.1$ ). The spectra have been scaled up for clarity: they overlap almost exactly.

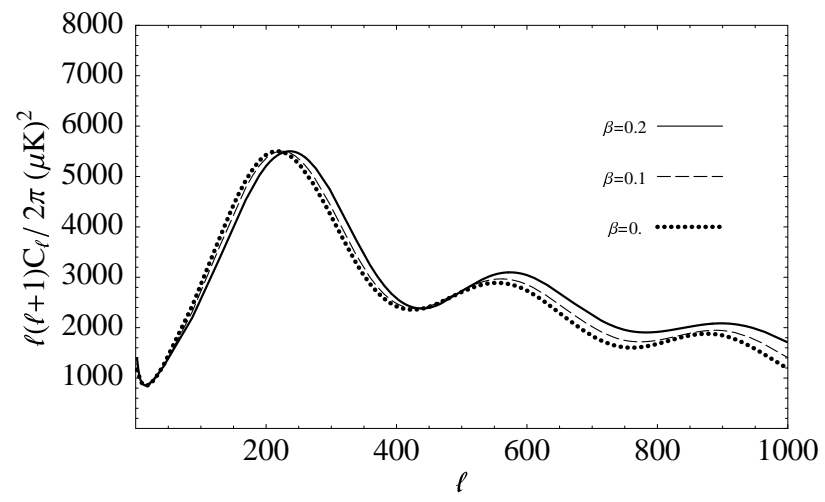

Figure 5. CMB spectra for increasing values of the coupling constant (the other parameters are $N=1.5, h=0.65, \omega_{b}=$ $\left.0.01, \omega_{c}=0.1\right)$. Notice that the peaks not only move to the right but also change in height.

\section{Constraining past and present equation of state with CMB}

Our theoretical model depends on three scalar field parameters and four cosmological parameters:

$$
\beta, \mu, N, n_{s}, h, \omega_{b}, \omega_{c}
$$

where $\omega_{b}=\Omega_{b} h^{2}$ and $\omega_{c}=\Omega_{c} h^{2}$. In Amendola et $a l$. (2002) we calculated the $C_{\ell}$ spectra by a modified CMBFAST (Seljak and Zaldarriaga 1996) code that includes the full set of coupled perturbation equations. We used as dataset the COBE data analyzed in Bond et al. (2000), and the high resolution data of Boomerang (Netterfield et al. 2002), Maxima (Lee et al. 2002), and DASI (Halverson et al. 2002). We adopted a pure log-normal likelihood; the overall amplitude and the calibration errors of Boomerang (10\%) and of Maxima and DASI (4\%) have been integrated out analytically. The theoretical spectra have been binned as the experimental ones. In order to compare with the previous analyses we assume uniform priors with the parameters confined in the range $\beta \in(0,0.3), \quad N \in(1,8.5)$, $n_{s} \in(0.7,1.3), h \in(0.45,0.9), \quad \omega_{b} \in(0.005,0.05)$, $\omega_{c} \in(0.01,0.3)$. The same age constraints $(>10 \mathrm{Gyr})$ used in most previous analyses is adopted here.

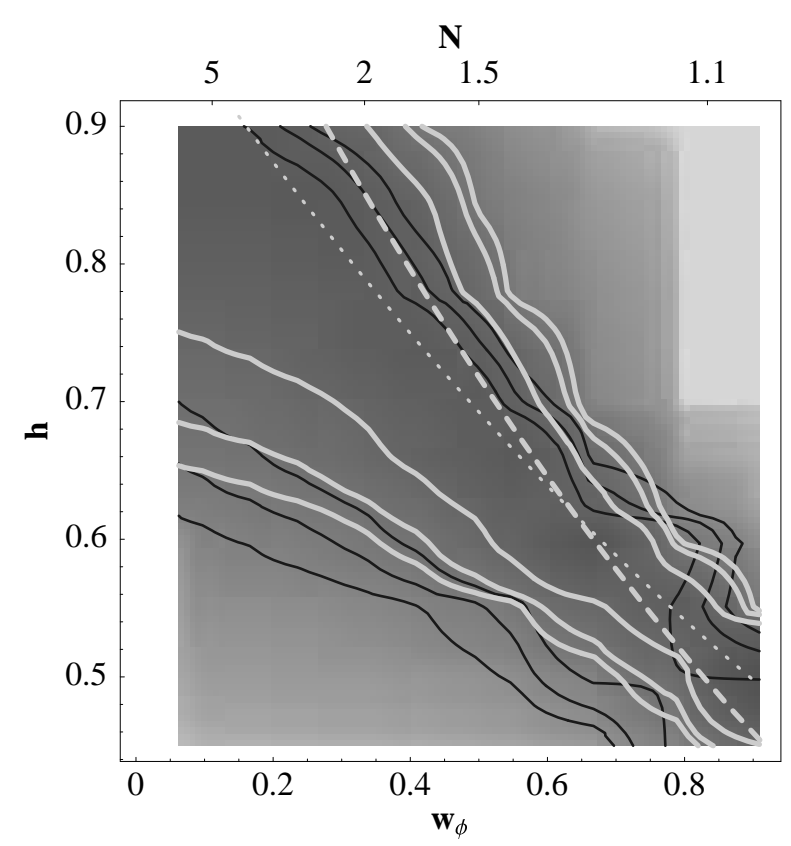

Figure 6. Likelihood contour plots in the space $N, h$ marginalizing over the other parameters at the 68,90 and 95\% c.l.. The white thick lines refer to the coupled case, the black curves to the uncoupled case. The dotted line is the likelihood degeneracy curve, the dashed line is the expected degeneracy curve. Notice that only fixing $h$ smaller than 0.65 it would be possible to exclude the cosmological constant at $95 \%$ c.l.

In Fig. 6 we show the likelihood curves for $N, h$ marginalizing over the remaining parameters. The contours of the likelihood plot follow the expected degeneracy of the angular diameter distance. The residual deviation from the expected degeneracy line is due to the age prior ( $i 10 \mathrm{Gyr})$ that favors small $h$ values. Notice that for $h>0.75$ no upper limit to $N$ can be given with the current CMB data no matter how precise $h$ is, and that only assuming $h<0.65$ it becomes possible to exclude at $95 \%$ c.l. the pure cosmological constant. In Fig. 7 we show the likelihood for all parameters, marginalizing in turn over the others. We find the following constraints at $95 \%$ c.l.:

$$
N>1.5, \quad \beta<0.16
$$

(the limit on $N$ corresponds to $\alpha<2$ ). The limit on $N$ is however only a formal one: the likelihood never vanishes in the definition domain and even $N=1$ (the exponential potential) is only a factor of five less likely than the peak and certainly cannot be excluded on this basis. Moreover, the lower bound on $N$ is prior-dependent: allowing smaller values of $h$ it would weaken. Clearly, if we adopt narrow priors on $h$, we can indeed obtain more stringent bounds on $N$, as shown in the same Fig. 7 . In contrast, the constraint on $\beta$ does 
not depend sensibly on the prior on $h$ and the likelihood for $\beta$ does vanish at large $\beta$. In place of $N$ and $\beta$ we can use as well the equation of state during tracking and during $\phi \mathrm{MDE}$, respectively, as likelihood variables, using Eqs. (13) and (14). Then we obtain at the $95 \%$ c.l.

$$
w_{\phi(\text { tracking })}<0.8, \quad 1<w_{e(\phi M D E)}<1.01
$$

This shows that the effective equation of state during $\phi \mathrm{MDE}$, i.e. between equivalence and tracking, is close to unity (as in a pure matter dominated epoch) to within one per cent. The striking difference between the level of the two constraints in (18) well illustrates the main point of this paper: the CMB is much more sensitive to the dark energy coupling than to its potential.
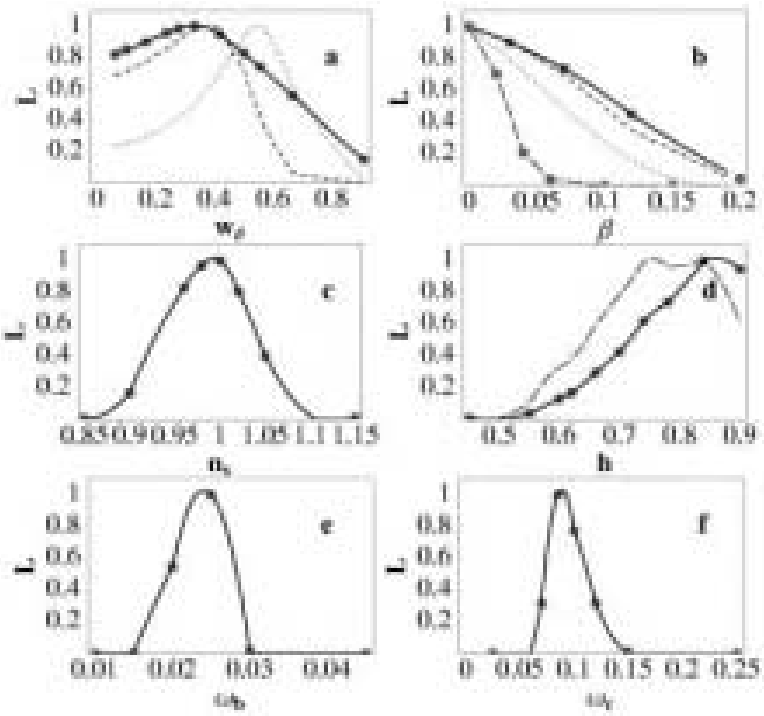

Figure 7. Marginalized likelihood for tracking trajectories. The equation of state in panel $a$ is $w_{\phi}=1 /(2 N-1)$. In panels $\mathrm{a}$ and $\mathrm{b}$ we plot as a dotted line the likelihood assuming $h=0.65 \pm 0.05$ and as dashed line $h=0.75 \pm 0.05$ (gaussian prior). In panel $b$ the long-dashed line is the Planck-like likelihood. In panel $d$ the long-dashed line is for $\beta=0$.

The other parameters are (we give here for simplicity the mean and the one sigma error, while the limit is at the $95 \%$ c.l.)

$$
\begin{gathered}
n_{s}=0.99 \pm 0.05, \quad \omega_{b}=0.023 \pm 0.004, \\
\omega_{c}=0.092 \pm 0.02, \quad h>0.62
\end{gathered}
$$

The total dark energy density turns out to be $\Omega_{\phi}>0.60$ (95\% c.l.). It appears that the limits on the cosmological parameters $n_{s}, \omega_{b}, \omega_{c}$ are almost independent of $\beta$, while a non-zero $\beta$ favors higher $h$ (Fig. 7 , panel $d$ ). It is interesting to compare with the current constraints from the Hubble diagram of the supernovae Ia, where one obtains quite a stronger bound on the equation of state, $w_{\phi}<0.4$ or $N>1.75$ at the same c.l..

Finally, in Fig. 7 (panel $b$ ) we plot the likelihood for $\beta$ that an experiment with no calibration uncertainty and limited only by cosmic variance, like the Planck mission (de Zotti et al. 1999), can achieve. We find $\beta<0.05$ (95\% c.l.) (using the specifications of the MAP satellite we find $\beta<0.1)$.

\section{Conclusion: the CMB as a gravity probe}

A dark matter-dark energy interaction would obviously escape any local gravity experiment: cosmological observations like the CMB are then the only way to observe such a phenomenon. Since observations require the baryons to be decoupled from dark energy (or coupled much more weakly than dark matter), the search for a non-zero $\beta$ is in fact also a test of the equivalence principle. We found that current CMB data are capable to put an interesting upper bound to the dark matter dark energy coupling:

$$
\beta<0.16
$$

(95\% c.l.) regardless of the potential (within the class we considered). This implies that the scalar gravity is at least $1 / \beta^{2} \approx 40$ times weaker than ordinary tensor gravity. As shown in e.g. Amendola (1999), the limit on $\beta$ can be restated as a limit on the constant $\xi$ of the non-minimally coupled gravity, defined by the gravity Lagrangian density

$$
L=\sqrt{-g}\left(1-\frac{1}{2} \xi \phi^{2}\right) R+L_{\phi}
$$

where $L_{\phi}$ is the canonical scalar field Lagrangian ( we are assuming that the Lagrangian for the baryon fields couples to a conformally related metric). Since for small $\xi$ one has $\xi \approx 2 \beta^{2} / 3$, we have $\xi<0.017$. Similarly, one can express the limit in terms of the original BransDicke parameter $\omega$ defined by the Lagrangian

$$
L=\sqrt{-g}\left(-\phi R+\frac{\omega}{\phi} \phi_{, \mu} \phi^{, \mu}\right)
$$

In this case we have $\omega>60$.

An experiment like the Planck mission can lower the upper bound to $\beta$ to 0.05 (95\% c.l.): scalar gravity would be in this case at least 400 times weaker than ordinary tensor gravity. This limit is comparable to those that local gravity experiments impose on the scalar gravity coupling to baryons, expressed in term of the "post-Einsteinian" (Groom et al. 2001) parameter $\eta=4 \beta^{2} / 3=-0.0007 \pm 0.0010$, i.e. $\beta_{\text {baryons }}^{2}<10^{-3}$ (see e.g. Groom et al. 2000).

In contrast, CMB data, on their own, cannot put any firm limit to the dark energy potential, unless a narrow prior on $h$ is adopted: e.g. $h=0.65 \pm 0.05$ gives $w_{\phi}=0.55 \pm 0.2$ while $h=0.75 \pm 0.05$ gives $w_{\phi}=0.35 \pm 0.2$ (assuming gaussian priors and marginalizing as usual over all the other parameters, including the coupling).

The nature of the dark energy is one of the most exciting and challenging issue of modern cosmology. Even 
assuming that the SNIa dimming is caused by some evolutionary effect, we are still confronted with the problem of reconciling a flat universe as seen by the CMB data with the small fraction of matter in clusters of galaxies. Lacking any definite suggestion as to its properties, cosmologists cannot do much better than trying to infer its nature from observations. We modeled the dark energy as a scalar field governed by a generic inverse power-law potential and a coupling to dark matter. The coupling introduces a new interaction that is unobservable on local scales but gives strong signatures for as concerns the perturbation growth and the proper distance. We reviewed the current constraints from the $\mathrm{CMB}$, finding that the coupling of the dark energy to dark matter cannot exceed 0.16 in terms of the strength ratio $\beta$. The future data from the Planck mission can reduce the upper limit to a level comparable to the limits that local gravity experiments put on the coupling to baryons.

\section{References}

[1] A. Albrecht, C.P. Burgess, F. Ravndal, and C. Skordis, astro-ph/0107573.

[2] J.S. Alcaniz and J. A. Lima, Astrophys. J. 521, L87 (1999).

[3] L. Amendola, Phys. Rev. D60, 043501 (1999).

[4] L. Amendola and D. Tocchini-Valentini, Phys. Rev. D 64, 043509 (2001).

[5] L. Amendola, Phys. Rev. D62, 043511 (2000).

[6] L. Amendola, Phys. Rev. Lett. 86, 196 (2001).

[7] L. Amendola and D. Tocchini-Valentini (2002) astro$\mathrm{ph} / 0111535$.

[8] L. Amendola, C. Quercellinni, D. Tocchini-Valentini, and A. Pasqui, (2002).

[9] C. Baccigalupi, F. Perrotta, and S. Matarrese, Phys. Rev. D 61, 023507 (2000).

[10] C. Baccigalupi, A. Balbi, S. Matarrese, F. Perrotta, and N. Vittorio, (2001).

[11] A. B. Batista, J. C. Fabris, and R. de Sa Ribeiro, Gen. Rel. Grav. 33, 1237 (2001).

[12] R. Bean and A. Melchiorri, astro-ph/0109097.

[13] O. Bertolami and P. J. Martins, Phys. Rev. D 61, 064007 (2000).

[14] A.P. Billyard and A.A. Coley, Phys. Rev. D61, 083503 (2000).

[15] J.R. Bond et al., Ap. J. 533, 19 (2000).

[16] R.R. Caldwell, R. Dave, and P.J. Steinhardt Phys. Rev. Lett. 80, 1582 (1998).

[17] M.O. Calvao, J.R.T. De Mello Neto, and I. Waga, astro-ph/0107029.

[18] X. Chen and M. Kamionkowsky, Phys. Rev. D60, 104036 (1999).

[19] T. Chiba, Phys. Rev. D60, 083508 (1999).

[20] T. Chiba, Phys. Rev. D64, 103503 (2001).
[21] L.P. Chimento, A. S. Jakubi, and D. Pavon, Phys. Rev. D62, 063508 (2000).

[22] A. R. Cooray and D. Huterer, Astrophys. J. 513, L95 (1999).

[23] E.J. Copeland, A.R. Liddle, and D. Wands, Phys. Rev. D57, 4686 (1997).

[24] P.S. Corasaniti and E. Copeland, astro-ph/0107378.

[25] T. Damour and K. Nordtvedt, Phys. Rev. Lett. 70, 2217 (1993)

[26] T. Damour, G. W. Gibbons, and C. Gundlach, Phys. Rev. Lett. 64, 123, (1990).

[27] T. Damour, F. Piazza, and G. Veneziano, hepth/0205111.

[28] G. de Zotti et al., (1999), AIP Conf. Proc. 476: 3K cosmology, 204, eds. L. Maiani, F. Melchiorri, N. Vittorio (Woodbury, N.Y. : American Institute of Physics)

[29] M. Doran, M. Lilley, and C. Wetterich, (2001) astro$\mathrm{ph} / 0105457$.

[30] M. Doran, M. J. Lilley, J. Schwindt, and C. Wetterich, Astrophys. J. 559, 501 (2001).

[31] G. Esposito-Farese and D. Polarsky, Phys. Rev. D63, 063504 (2001).

[32] V. Faraoni, Phys. Rev D62, 023504 (2000).

[33] J. Frieman, C.T. Hill, A. Stebbins, and I. Waga, Phys. Rev. Lett. 75, 2077 (1995).

[34] M. Gasperini, F. Piazza and G. Veneziano, (2001) grqc/0108016.

[35] F. Giovi, F. Occhionero, and L. Amendola, Mon. Not. Roy. Astron. Soc. 325, 1097 (2001).

[36] D.E. Groom et al. Eur. Phys. J. C 15, 1 (2000).

[37] N.W. Halverson et al., (2001) astro-ph/0104489.

[38] D.J. Holden and D. Wands, Phys. Rev. D61, 043506 (2000).

[39] R. Horvat, astro-ph/0007168.

[40] G. Huey, L. Wang, R. Dave, R.R. Caldwell, and P.J. Steinhardt, Phys. Rev. D59, 063005 (1999).

[41] R. Jimenez and A. Loeb, arXiv:astro-ph/0106145.

[42] A.T. Lee et al., Ap. J. 561, L1 (2001).

[43] M. Li, X. Wang, B. Feng, and X. Zhang astro$\mathrm{ph} / 0112069$.

[44] C.B. Netterfield et al., (2001) astro-ph/0104460.

[45] J. Newman, C. Marinoni, A. Coil and M. Davis, astroph/0109131.

[46] S. Perlmutter et al. Ap.J. 517, 565 (1999).

[47] M. Pietroni, (2002) hep-ph/0203085.

[48] B. Ratra B. and P.J.E. Peebles, Phys. Rev. D37, 3406 (1988).

[49] A.G. Riess et al. A.J., 116, 1009 (1998).

[50] A.A. Sen and S. Sen, MPLA, 16, 1303 (2001).

[51] Seljak and M. Zaldarriaga, Ap. J. 469, 437 (1996).

[52] P.J. Steinhardt, Limin Wang, Ivaylo Zlatev, Phys. Rev. D59, 123504 (1999). 
[53] D. Tocchini-Valentini and L. Amendola, Phys. Rev. D65, 063508 (2002).

[54] J.P. Uzan, Phys. Rev. D59, 123510 (1999).

[55] D. Wands, E.S. Copeland, and A. Liddle, Ann. N.Y.
Acad. Sci. 688, 647 (1993).

[56] C. Wetterich, Nucl. Phys. B 302, 668 (1998).

[57] C. Wetterich, A\& A, 301, 321 (1995). 